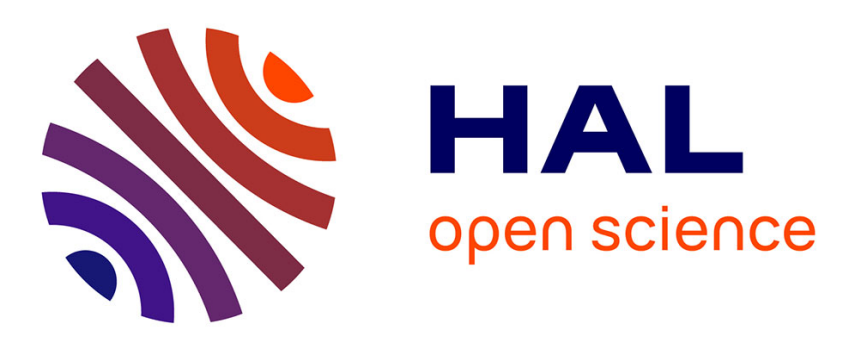

\title{
Identification of multi-core interference
}

Frédéric Boniol, Claire Pagetti, Nathanaël Sensfelder

\section{To cite this version:}

Frédéric Boniol, Claire Pagetti, Nathanaël Sensfelder. Identification of multi-core interference. 19th International Symposium on High Assurance Systems Engineering (HASE 2019), Jan 2019, Hangzou, China. 10.1109/HASE.2019.00024 . hal-02441353

\section{HAL Id: hal-02441353 \\ https://hal.science/hal-02441353}

Submitted on 15 Jan 2020

HAL is a multi-disciplinary open access archive for the deposit and dissemination of scientific research documents, whether they are published or not. The documents may come from teaching and research institutions in France or abroad, or from public or private research centers.
L'archive ouverte pluridisciplinaire HAL, est destinée au dépôt et à la diffusion de documents scientifiques de niveau recherche, publiés ou non, émanant des établissements d'enseignement et de recherche français ou étrangers, des laboratoires publics ou privés. 


\title{
Identification of multi-core interference
}

\author{
Frédéric Boniol Claire Pagetti Nathanaël Sensfelder
}

November 22, 2018

\begin{abstract}
The CAST-32A provides some guidelines to help certify multi-core-based systems in the avionics domain. One major requirement is to compute all the potential interference and to provide adequate mitigation means. In this paper, we compare two approaches to identify the interference: the initiatortarget and the PHYLOG models. The latter is more compact and efficient, despite also covering all of the problematic conflictual situations.
\end{abstract}

Keywords: Multi-core, certification, timing interference

\section{Introduction}

The last decade has seen the emergence of multi-core processors, i.e. chips integrating several cores linked by a shared interconnect. Although these architectures have been shown to provide huge gains in performance, they have severe lapses in time predictability [20, 21, one of the key elements of certification expectations.

\subsection{Identification of potential interference}

Aeronautic certification authorities, in association with industrial manufacturers, have published the Multi-Core Certification Review Item (MCP-CRI) 11] (also published as the CAST-32A position paper 7]), in order to provide a set of guidances for software planning and verification on multi-core chips.

Due to resource sharing, couplings exist at the platform level. These can cause interference between applications, which, in turn, may lead to unexpected delays, and even the alteration or loss of data. These three issues are not acceptable in the aeronautics domain and must thus be avoided. In terms of certification, this entails a four steps process: First, the applicant must identify all interference channels. In the CAST-32A terminology, an interference channel is a platform property that may cause interference between independent applications. Second, the applicant must classify the interference as either acceptable, tolerable, or unacceptable. Third, for each unacceptable interference, they must provide a mean of mitigation to prevent the system from having catastrophic behaviors. In that context, mitigation signifies that some mechanisms have been proposed to forbid unacceptable interference or reduce their effect to acceptable or tolerable levels. For example, if a resource being accessed in parallel by more than two requesters would lead to a non-acceptable delay, mitigation could take the form of a run-time mechanism that sequentializes the access. Fourth and final, the applicant must argue why the means of mitigation are adequate and why unacceptable interference will never occur during aircraft operations.

This requirement is called resource usage 3 in the CAST-32A. In the sequel, we will only focus on this particular objective and, more precisely, on the identification of interference.

\subsection{Objectives and contribution}

To the best of our knowledge, few works have proposed solutions for resource usage 3. Researchers from Thales have proposed the Initiator-Target Model [6, 14, 17, 18] to help identify the interference channels on multi-core chips. Their model is very simple, but suffers from a combinatorial explosion.

PHYLOG is a French project (2016-2020), funded by the French civil aeronautic agency (DGAC), which aims at offering a model-based and software-aided certification framework for aeronautics systems based on multi/many-core architectures. In [3], we have defined the premises of the PHYLOG model, presented the notions of interference channels and transactions, and shown an automated process to find the interference channels through the use of WEIRD [4]. 
The objective of this paper is to compare and link the initiator-target and PHYLOG models. For that purpose, we start with a formal definition of the initiator-target model (see section 2). We then refine and formalize our former definition of interference channels (see section 3). We then show that our representation is more compact than the initiator-target model despite remaining as expressive. Indeed, our interference channels are the representative elements of the equivalence classes of an equivalence relation (see section 4). All our formalization and computation are supported by implementations made in IDP [10] (see section 5), used as a replacement for WEIRD [4].

\section{The initiator-target model}

The initiator-target model has been introduced in [6] and reused in [14, 17], and [18. The goal was to provide a theoretical view for the identification of the interference channels (called performance contentions in [6]) that can occur in a multi-core processor.

\section{$2.1 \quad$ Overview}

According to their definition, a multi-core is composed of three types of components:

- Smart initiator components, i.e. components which can initiate single transactions through the architecture to target components. Processing cores (CPU) are examples of smart initiator components. They can, for instance, initiate memory access transactions to memory controllers.

- Non smart initiator components, i.e. initiator components which can only initiate dual transactions (i.e., with two targets at the same time). DMA are examples of non smart initiator components.

- Target components, i.e. end-components targeted by smart or non smart initiators.

Intermediate components (such as internal buses) between initiators and targets are simply ignored.

Example 1 Let us illustrate the initiator-target model on the simple architecture shown in Figure 1 . composed of two smart initiators (the two CPUs), two non smart initiators (the two DMAs), and four targets (the two memory controllers, the PCIe controller, and one L3 cache used as SRAM memory).

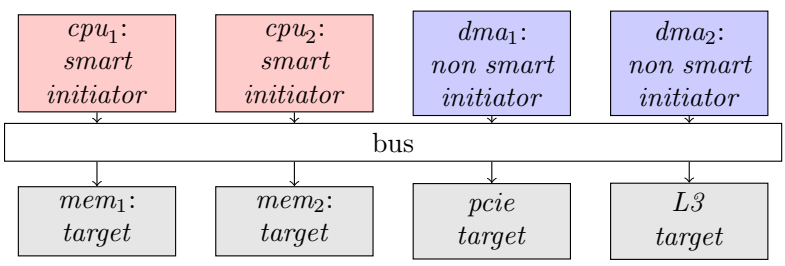

Figure 1: Architecture $P_{1}$

Notations 1 For the sake of readability, our figures use the following color code: smart initiators are in red, non smart initiators are in blue, targets are in gray, and other components are in white.

An interference channel is seen as a combination of single test classes (or single test cases) where an interference occurs. A single test class is a transaction initiated by an initiator and targeting a target (for smart initiators) or two targets (in case of non smart initiators).

Example 2 In the architecture depicted Figure 1, cpu $u_{1} \rightsquigarrow m_{1} m_{1}, c p u_{2} \rightsquigarrow m_{2}$, and pcie $\rightsquigarrow d m a_{1} \rightsquigarrow$ mem $_{2}$ are examples of single test classes. The first two may denote either read or write transactions from

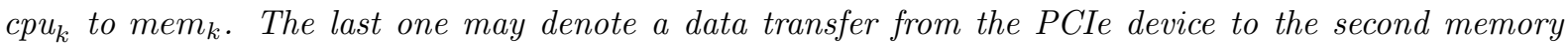
through the $d m a_{1}$.

$$
\left(c p u_{1} \rightsquigarrow m e m_{1}\right)\left\|\left(c p u_{2} \rightsquigarrow m e m_{2}\right)\right\|\left(\text { pcie } \rightsquigarrow d m a_{1} \rightsquigarrow m e m_{2}\right)
$$

is a test class composed of three single test classes running in parallel. 


\section{$2.2 \quad$ Formalization}

Let us now introduce a set-based formalization of the initiator-target model.

Definition 1 (Initiator-target model) In the initiator-target model, an architecture $P$ is defined by $P=(\mathcal{C}, \rightarrow)$ where

- $\mathcal{C}=S I \cup N S I \cup T \cup O$ with $S I$ being the set of smart initiators, NSI the set of non smart initiators, $T$ the set of targets, and $O$ the set of other components. All those sets are disjoint. In the sequel, we will note $n_{S I}=\operatorname{card}(S I), n_{N S I}=\operatorname{card}(N S I)$ and $n_{T}=\operatorname{card}(T)$;

- $\rightarrow \subseteq \mathcal{C} \times \mathcal{C}$ are the hardware connections between components.

Definition 2 (Single test class) For an architecture $P=(\mathcal{C}, \rightarrow)$, a single test class for a smart initiator is a pair $(i, t) \in S I \times T$ such that there exists a path in $P$ from $i$ to $t$, i.e. $i \rightarrow^{*} t$. In the sequel, we write indifferently $(i, t)$ or $i \rightsquigarrow t$.

A single test class for a non smart initiator is a triplet $\left(i, t_{1}, t_{2}\right) \in N S I \times T \times T$ such that there exist a path in $P$ from $i$ to $t_{2}$ and one to $t_{1}$, i.e. $i \rightarrow^{*} t_{2}$ and $i \rightarrow^{*} t_{1}$. In the sequel, we write indifferently $\left(i, t_{1}, t_{2}\right)$ or $t_{1} \rightsquigarrow i \rightsquigarrow t_{2}$.

Definition 3 (Test classes) Let $P=(\mathcal{C}, \rightarrow)$ be an architecture. A test class is a set of $n$ single test classes and disjoint initiators. For instance, a test class $t_{c}$ of size 2 is of form $t_{c}=\left\{\left(i_{1}, t_{1}\right),\left(i_{2}, t_{2}\right)\right\}$ or $t_{c}=\left\{\left(i_{1}, t_{1}\right),\left(i_{2}, t_{2}, t_{3}\right)\right\}$ or $t_{c}=\left\{\left(t_{1}, i_{1}, t_{2}\right),\left(i_{2}, t_{3}, t_{4}\right)\right\}$ with $i_{1} \neq i_{2}$. In the sequel, we write indifferently $\left\{\left(i_{1}, t_{1}\right),\left(i_{2}, t_{2}\right)\right\}$ or $\left(i_{1} \rightsquigarrow t_{1}\right) \|\left(i_{2} \rightsquigarrow t_{2}\right)$.

An interference channel is a test class composed of 2 or more single test classes.

Definition 4 Let $P=(\mathcal{C}, \rightarrow)$ be an architecture. Let us note $T C_{\infty}^{P}$ the set of test classes and $T C_{n}^{P}$ those of size $n$ :

$$
T C_{\infty}^{P}=\bigcup_{n=1}^{n_{S I}+n_{N S I}} T C_{n}^{P}
$$

Proposition 1 (Total number of test classes [6]) The number of all possible test classes of $P$ is:

$$
\operatorname{card}\left(T C_{\infty}^{P}\right)=\left(1+n_{T}\right)^{n_{S I}} \cdot\left(1+n_{T}^{2}\right)^{n_{N S I}}-1
$$

Example 3 Let us once again consider the architecture $P_{1}$ shown in Figure 1: $n_{S I}=2, n_{N S I}=2$,

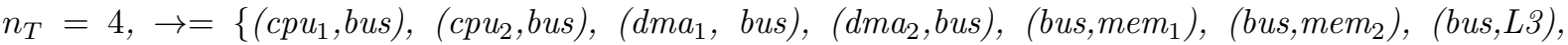
(bus,pcie)\}. Applying equation 1 yields:

$$
\operatorname{card}\left(T C_{\infty}^{P_{1}}\right)=(1+4)^{2} \cdot\left(1+4^{2}\right)^{2}-1=7224
$$

meaning that the interference analysis may need up to 7224 test classes to be analyzed on this architecture. Aeronautic certification standards require the assessment of the worst case execution time (WCET) for the critical software functions running on the cores of the processor. As stated in the introduction, interference may strongly affect this execution time. It is up to the designer to characterize the severity of each interference with respect to the execution time of each software function, and, at the end, to show that all the unacceptable interference (i.e., the ones that induce too high WCETs) are properly mitigated by appropriate means (e.g., arbiters, time-triggered execution schemes, etc.). In the case of the (rather small) architecture $P_{1}$, such an assessment requires the investigation of the 7224 test classes.

\subsection{Hypotheses}

In the seminal paper, there was no specific rule about the reachability of a target by an initiator. Implicitly, the authors assumed that all targets were reachable by all initiators. Moreover, they did not make a distinction on the type of transactions (e.g. read or write). Finally, they assumed a unique path from any given initiator to any given target, which is not the case in many-cores. Most commercial multi-core processors satisfy these two hypotheses. Let us formalize them:

- Hyp1. All targets are reachable by all initiators: $\forall i \in S I \cup N S I, \forall t \in T, i \rightarrow^{*} t$

- Hyp2. There is a unique path from an initiator to any non initiator component: $\forall i \in S I \cup N S I, \forall t \in$ $O \cup T$, if $i \rightarrow a_{1} \rightarrow \ldots \rightarrow a_{n} \rightarrow t$ and $i \rightarrow b_{1} \rightarrow \ldots \rightarrow b_{m} \rightarrow t$, then $n=m$ and $\forall k, a_{k}=b_{k}$

Proposition 2 The reachability relation $\rightarrow$ satisfying Hyp2 is acyclic and defines a partial order $<_{P}$ : $\forall \alpha, \beta \in S I \cup N S I \cup T \cup O, \alpha<_{P} \beta \Leftrightarrow \alpha \rightarrow^{*} \beta$. 


\section{PhYLOG model}

In the PHYLOG project, we need to tackle the identification of all interference channels as in Brindejonc et al.'s approach.

\subsection{Overview}

We believe that the current initiator-target model is insufficient as is and that it must be enriched.

1. Brindejonc's approach is a black-box approach: it does not consider internal components. Two architectures with different topologies may be characterized by the same test classes, even when their interference differ.

2. Test classes do not necessarily lead to any actual interference between transactions. For instance, in the architecture $P_{2}$ depicted Figure 2 the test class $\left(c p u_{1} \rightsquigarrow m e m_{1}\right) \|\left(p c i e \rightsquigarrow d m a_{1} \rightsquigarrow L 3\right)$ does not cause any contention as these transactions cross two different buses in parallel without interfering with each other. Many other test classes in $P_{2}$ are interference-free as well.

3. Their approach is simple, but suffers from scalability issues: For a T4240 processor, composed of 12 cores $\left(n_{S I}=12\right), 3$ DMAs $\left(n_{N S I}=3\right), 2$ memory controllers, 1 PCIe, and 1 L3 cache used as SRAM memory $\left(n_{T}=4\right)$, there are more than $10^{12}$ test cases.

Ideally, the number of test classes to be explored should be as low as possible. We thus propose grouping them according to the interference they cause on the components.

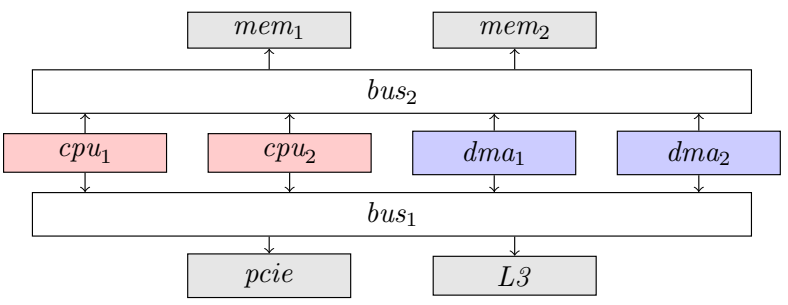

Figure 2: Architecture $P_{2}$

\subsection{Transaction model}

Definition 5 (Transaction) For an architecture $P=(\mathcal{C}, \rightarrow)$, a transaction is a finite branching word of components

$$
\operatorname{tr}=i \cdot\left(\left(b_{1} \ldots b_{n}\right)+\left(c_{1} \ldots c_{m}\right)\right)
$$

with $i \in S I \cup N S I$; for all $j, b_{j} \in O \cup T,\left(b_{j}, b_{j+1}\right) \in \rightarrow$ and $\left(i, b_{1}\right) \in \rightarrow$. If $i \in S I, c_{1} \ldots c_{m}=\epsilon$ is the empty word; otherwise, for all $j, c_{j} \in O \cup T,\left(c_{j}, c_{j+1}\right) \in \rightarrow$ and $\left(i, c_{1}\right) \in \rightarrow$.

In the following, we will consider that the branching operator " + " is commutative. That is, $t r=$ $i \cdot\left(\left(b_{1} \ldots b_{n}\right)+\left(c_{1} \ldots c_{m}\right)\right)=i \cdot\left(\left(c_{1} \ldots c_{m}\right)+\left(b_{1} \ldots b_{n}\right)\right)$.

Example 4 A single test class is a transaction from an initiator to one or two targets. In $P_{1}$, the single test class $\mathrm{cpu}_{1} \rightsquigarrow \mathrm{mem}_{1}$ is the transaction $\mathrm{cpu}_{1}$. (bus.mem $\left.\mathrm{m}_{1}+\epsilon\right)$; whereas pcie $\rightsquigarrow \mathrm{dma} \rightsquigarrow \mathrm{mem}_{2}$ is the transaction dma.(bus.mem ${ }_{2}+$ bus.pcie).

Definition 6 We define a series of useful functions for a transaction $\operatorname{tr}=i \cdot\left(\left(a_{1} \ldots a_{n} \cdot b_{1} \ldots b_{m}\right)+\right.$ $\left.\left(a_{1} \ldots a_{n} \cdot c_{1} \ldots c_{p}\right)\right)$ with for all $l, k b_{l} \neq c_{k}$ :

- $h d(t r)=i$ (head of the transaction),

- $\operatorname{prefix}(t r)=a_{1} \ldots a_{n}$ (common prefix of the transaction, possibly empty).

- $\operatorname{proj}_{1}(t r)=i \cdot a_{1} \ldots a_{n} \cdot b_{1} \ldots b_{m}$ (first branch of the transaction),

- $\operatorname{proj}_{2}(t r)=i \cdot a_{1} \ldots a_{n} \cdot c_{1} \ldots c_{p}$ (second branch), 
- $\lg (\mathrm{tr})=\left\{a_{1}, \ldots, a_{n}, b_{1}, \ldots, b_{m}, c_{1}, \ldots, c_{m}\right\}$ (language of the transaction, i.e., the set of components involved),

- $t l\left(\alpha_{1} \ldots \alpha_{n}\right)=\alpha_{n}$ (tail of a word),

- $\operatorname{pred}\left(\alpha_{1} \ldots \alpha_{n-1} \cdot \alpha_{n}\right)=\alpha_{n-1}$ (penultimate component of a word).

According to hypothesis Hyp2, each $t c$ is modeled by a unique transaction $t r$ in the PHYLOG model.

Definition 7 (Transactions associated to a single test class) When $(i, t) \in S I \times T$, the associated PHYLOG transaction is defined as phy $(i, t)=\operatorname{tr}$ with $h d(t r)=i, t l\left(\operatorname{proj}_{1}(t r)\right)=t$ and $\left.\operatorname{proj}_{2}(t r)\right)=\epsilon$.

When $\left(i, t_{1}, t_{2}\right) \in N S I \times T \times T$, the associated PHYLOG transaction is defined as phy $\left(i, t_{1}, t_{2}\right)=t r$ with $h d(t r)=i, t l\left(\operatorname{proj}_{1}(t r)\right)=t_{1}$ and $t l\left(\operatorname{proj}_{2}(t r)\right)=t_{2}$.

Proposition 3 Let $P=(\mathcal{C}, \rightarrow)$ be an architecture satisfying Hyp1 and Hyp2. Let $\operatorname{tr}=i \cdot\left(\left(b_{1} \ldots b_{n}\right)+\right.$ $\left.\left(c_{1} \ldots c_{m}\right)\right)$ be a transaction in $P$. Then:

1. $\forall j \neq k, b_{j} \neq b_{k}$ (a component can appear at most once in the b branch),

2. either $\left(c_{1} \ldots c_{m}\right)=\epsilon$ (in the case of a smart initiator) or $\forall j \neq k, c_{j} \neq c_{k}$ (same as with the $b$ branch),

3. if there exist $j, k$ such that $b_{j}=c_{k}$, then $j=k$ and $\forall l<k, b_{l}=c_{l}$ (either the two branches do not share any component, or they share a common prefix).

4. $\forall l, l^{\prime}>\max \left\{j \mid b_{j}=c_{j}\right\}, b_{l} \neq c_{l^{\prime}}$ (after the common prefix, if it exists, the two branches do not share any component).

Proof 1 The first two points come from the acyclic property of the reachable relation $\rightarrow$. The third point is a consequence of hypothesis Hyp2. Let us suppose that there exists a common component $\alpha$ in the two branches $\left(\exists j, l, \alpha=b_{j}=c_{l}\right)$, then because of the unicity of the path from $i$ to $\alpha$, we have $\left(b_{1} \ldots b_{j}\right)=\left(c_{1} \ldots c_{l}\right)$. Thus, $j=l$ and $b_{k}=c_{k}$ for all $k<l$. The fourth point then comes immediately.
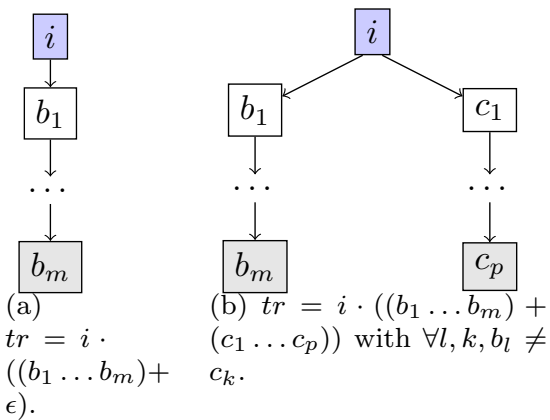

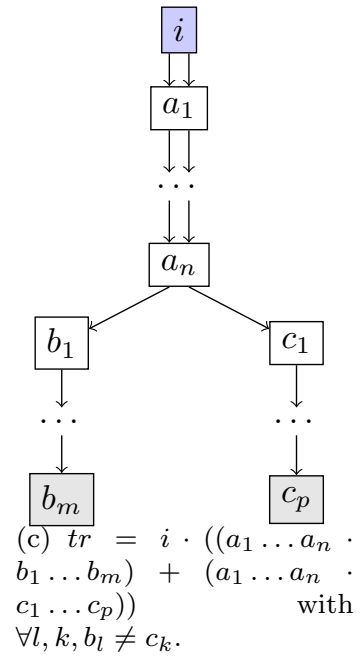

Figure 3: Transaction model

According to the proposition 3, a transaction can be graphically represented by the figure 3 . Figure 3(a) shows a transaction made by a smart initiator. Figure 3(b) describes a transaction made by a non smart initiator without a common prefix. Figure 3(c) describes a transaction made by a non smart initiator with a common prefix. After the prefix, all the components are different. Note that the transaction Figure $3(\mathrm{~b})$ is a particular case of Figure 3(c) in which the prefix word is empty: $\left(a_{1} \ldots a_{n}\right)=\epsilon$. 


\subsection{Test classes revisited - Truncated transactions}

A test class, as introduced by definition 3 , is a set of single test classes $t c_{1}, \ldots, t c_{n}$ issued by $n$ different initiators.

Definition 8 Let $t c=\left\{\left(i_{1}, t_{1}\right) \ldots\left(i_{m}, t_{m}, t_{m}^{\prime}\right)\right\}$ be a test class, the associated PHYLOG transactions are $p h y(t c)=\left\{\operatorname{tr}_{1}, \ldots, t r_{m}\right\}=\left\{p h y\left(i_{1}, t_{1}\right), \ldots, p h y\left(i_{m}, t_{m}, t_{m}^{\prime}\right)\right\}$.

The shared components in a set of transactions are then:

Definition 9 Let $t c=\left\{t r_{1}, \ldots, t r_{n}\right\}$ be a set of transactions, the language associated to tc is defined by: $l g(t c)=l g\left(t r_{1}\right) \cup \ldots \cup l g\left(t r_{n}\right)$ and shared $(t c)$ is the set of components involved in all transactions:

$$
\operatorname{shared}(t c)=\lg \left(\operatorname{tr}_{1}\right) \cap \ldots \cap \lg \left(\operatorname{tr}_{n}\right)
$$

We define the PHYlog test classes as the sets of truncated transactions where the interference-free parts of transactions are translated as the empty word $\epsilon$.

Definition 10 (Phylog test class) A Phylog test class is a set of $n$ transactions

$$
p t c=\left\{t r_{1}, \ldots, t r_{n}\right\}
$$

such that $\exists$ tc $=\left\{\left(i_{i}, t_{i}\right) \ldots\left(i_{m}, t_{m}\right)\left(i_{m+1}, t_{m+1}, t_{m+1}^{\prime}\right) \ldots\left(i_{n}, t_{n}, t_{n}^{\prime}\right)\right\} \in T C_{\infty}^{P}$ with

- $\forall k, h d\left(t r_{k}\right)=i_{k}$;

- $\forall j \leq m, t r_{j}=\operatorname{trunc}\left(t c, p h y\left(i_{j}, t_{j}\right)\right)$ where $\operatorname{trunc}\left(t c, i \cdot\left(a_{1} \ldots a_{n}+\epsilon\right)\right)=i \cdot\left(a_{1} \ldots a_{p}+\epsilon\right)$ with $p \leq n$ and $a_{p} \in \operatorname{shared}(t c)$;

- $\forall m+1 \leq j \leq n, \exists k \leq 3, \operatorname{tr}_{j}=\operatorname{trunc}_{k}\left(t c, p h y\left(i_{j}, t_{j}, t_{j}^{\prime}\right)\right)$ where

$$
\begin{gathered}
\operatorname{trunc}_{1}\left(t c, i \cdot\left(a_{1} \ldots a_{n}+b_{1} \ldots b_{m}\right)\right)=i \cdot\left(a_{1} \ldots a_{p}+a_{1} \ldots a_{p}\right) \\
\quad \text { with } p \leq n \text { and } a_{p} \in \operatorname{shared}(t c) \text { and } \forall k \leq p, b_{k}=a_{k} \\
\operatorname{trunc}_{2}\left(t c, i \cdot\left(a_{1} \ldots a_{n}+b_{1} \ldots b_{m}\right)\right)=i \cdot\left(a_{1} \ldots a_{p}+\epsilon\right) \\
\quad \text { with } p \leq n \text { and } a_{p} \in \operatorname{shared}(t c) \text { and } b_{p} \neq a_{p} \text { and } \forall k, b_{k} \notin \operatorname{shared}(t c) \\
\operatorname{trunc}_{3}\left(t c, i \cdot\left(\begin{array}{l}
\left.\left.a_{1} \ldots a_{n}+b_{1} \ldots b_{m}\right)\right)=i \cdot\left(a_{1} \ldots a_{p}+b_{1} \ldots b_{l}\right) \\
\text { with } p, l \leq n \text { and } a_{p} \in \operatorname{shared}(t c) \text { and } b_{l} \in \operatorname{shared}(t c)
\end{array}\right.\right.
\end{gathered}
$$

We note Trans the set of PHYLOG test classes.

Notations 2 When handling truncated transactions generated by smart initiators, we will deliberately remove the second branch which is empty. For instance, $\mathrm{cpu}_{1}$. bus stands for $\mathrm{cpu}_{1}$. $\left(\mathrm{bus}_{\mathrm{s}}+\epsilon\right)$. This contraction cannot be applied to truncated transactions generated by non smart initiators, as it would then be impossible to differentiate the prefix part.

Example 5 In $P_{1}, p t c=\left\{\mathrm{cpu}_{1}\right.$.bus, $\mathrm{cpu}_{2}$.bus $\} \in \mathbb{T}$ rans. Indeed, $\mathrm{cpu}_{k}$. bus can be obtained as the truncation of $\mathrm{cpu}_{k} \cdot($ bus.pcie $\left.+\epsilon)\right)$.

In architecture $P_{2}, p t c=\left\{\operatorname{cpu}_{1}\right.$. bus ${ }_{1}$, dma $_{1} .\left(\right.$ bus $\left.\left._{1}+\epsilon\right)\right\} \in \mathbb{T}$ rans. Indeed, let $t c=\left\{\left(\mathrm{cpu}_{1}, \mathrm{pcie}\right),\left(\mathrm{dma}_{1}, \mathrm{pcie}, \mathrm{mem}_{1}\right)\right\}$ then $\operatorname{cpu}_{1} \cdot$ bus $_{1}=\operatorname{trunc}\left(t c, \mathrm{cpu}_{1} \cdot\left(\right.\right.$ bus $\left.\left._{1} \cdot \operatorname{pcie}+\epsilon\right)\right)$ and $\operatorname{dma}_{1} \cdot\left(\right.$ bus $\left._{1}+\epsilon\right)=\operatorname{trunc}_{2}\left(t c\right.$, dma $_{1} \cdot\left(\right.$ bus $_{1} \cdot$ pcie + $\left.\left.\operatorname{bus}_{2} \cdot \mathrm{mem}_{1}\right)\right)$.

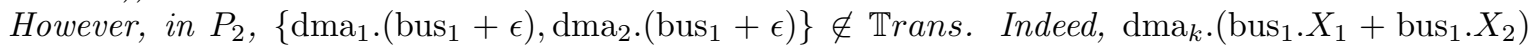
with $X_{j} \in\{$ pcie, L3 $\}$ can only be truncated with trunc ${ }_{1}$ as dma $_{k}$. $\left(\right.$ bus $_{1}+$ bus $\left._{1}\right)$. And the test case $\left\{\right.$ dma $_{1} \cdot\left(\operatorname{bus}_{1} \cdot X_{1}+\operatorname{bus}_{2} \cdot Y_{2}\right)$, dma $\left.2 .\left(\operatorname{bus}_{1} \cdot X_{2}+\operatorname{bus}_{2} \cdot Y_{2}\right)\right\}$ with $X_{j} \in\{$ pcie, L3 $\}$ and $Y_{j} \in\left\{\operatorname{mem}_{1}, \operatorname{mem}_{2}\right\}$ can only be truncated by trunc $_{3}$ as $\mathrm{dma}_{k}$. (bus $\left.{ }_{1}+\mathrm{bus}_{2}\right)$.

On the contrary, $p t c=\left\{\mathrm{cpu}_{1} \cdot\right.$ bus $_{1}$, dma $\mathrm{dm}_{1} \cdot\left(\right.$ bus $\left._{1}+\epsilon\right)$, dma $2 .\left(\right.$ bus $\left.\left._{1}+\epsilon\right)\right\} \in \mathbb{T}$ rans. Indeed, the completion

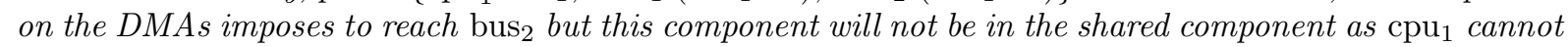
reach it.

\subsection{Interference channel}

We can now formally define the notion of interference channel. We distinguish two kinds of interference channels: the 1-interference channels which involve a single shared component, and the 2-interference channels which involve two shared components. 


\subsubsection{1-Interference channels}

Definition 11 (1-Interference channel) For an architecture $P=(\mathcal{C}, \rightarrow)$, a 1-interference channel is defined as

$$
\left(c, \bigcup_{n} T R_{1}^{n}\right)
$$

with $c \in O \cup T$ and

$$
T R_{1}^{n}=\bigcup\left\{\begin{array}{c}
\left\{r_{1}, \ldots, t r_{n}\right\} \in \mathbb{T} \text { rans } \mid \\
\forall i, t l\left(\operatorname{proj}_{1}\left(t r_{i}\right)\right)=c \wedge t l\left(\operatorname{proj}_{2}\left(t r_{i}\right)\right)=\{c, \epsilon\} \\
\wedge \forall i \neq j \\
h d\left(t r_{i}\right) \neq h d\left(t r_{j}\right) \\
\wedge \operatorname{pred}\left(\operatorname{proj}_{1}\left(t r_{i}\right)\right) \neq \operatorname{pred}\left(\operatorname{proj}_{1}\left(t r_{j}\right)\right)
\end{array}\right\}
$$

An interference channel ends with a shared component $c$. What happens after is irrelevant, as serialization occurs at that point and the transactions do not interfere with each other later on. We apply the same reasoning as in network calculus [16] when packets share a common path on several switches. This is known as the pay burst only once rule. Thus, interference only occurs on the first component shared by the $n$ transactions. Note that this could be improved in several ways, such as by considering the component entailing the worst case delay instead of simply taking the first one, or by grouping successive components into a super-component.

Example 6 Let us again consider the $P_{1}$ of Figure 1. There is a unique 1-interference channel with 11 combinations of transactions capable of occurring on the bus:

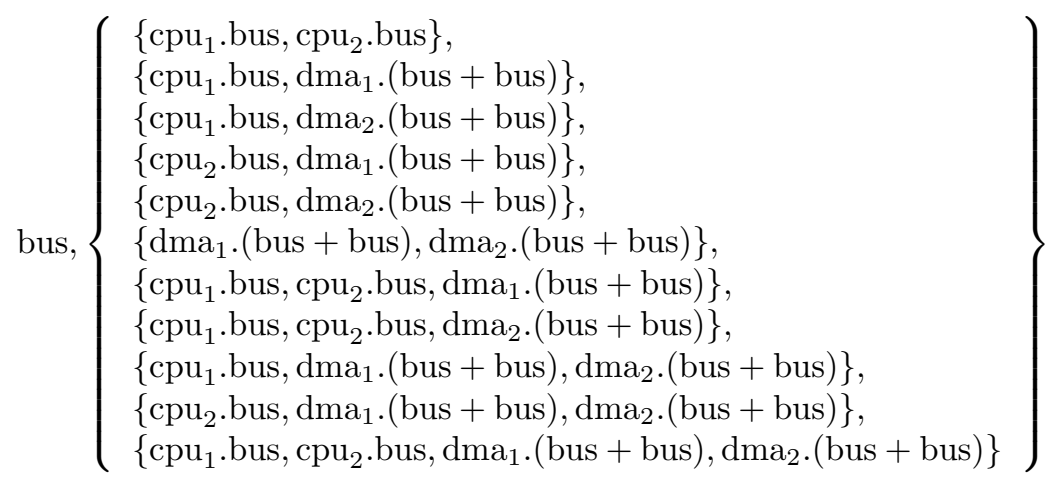

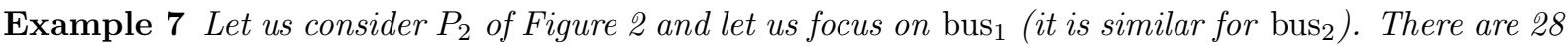
combinations of transactions. The interesting parts are those featuring the dma. Either the second branch of the transaction reaches bus ${ }_{1}$, or it is pruned (and replaced by $\epsilon$ ). The notation $\operatorname{dma}_{2} .\left(\operatorname{bus}_{1}+\left\{\right.\right.$ bus $\left._{1}, \epsilon\right\}$ ) represents the two transactions $\mathrm{dma}_{2} \cdot\left(\mathrm{bus}_{1}+\mathrm{bus}_{1}\right)$ and $\mathrm{dma}_{2} \cdot\left(\mathrm{bus}_{1}+\epsilon\right)$ in order to reduce the length of the table below.

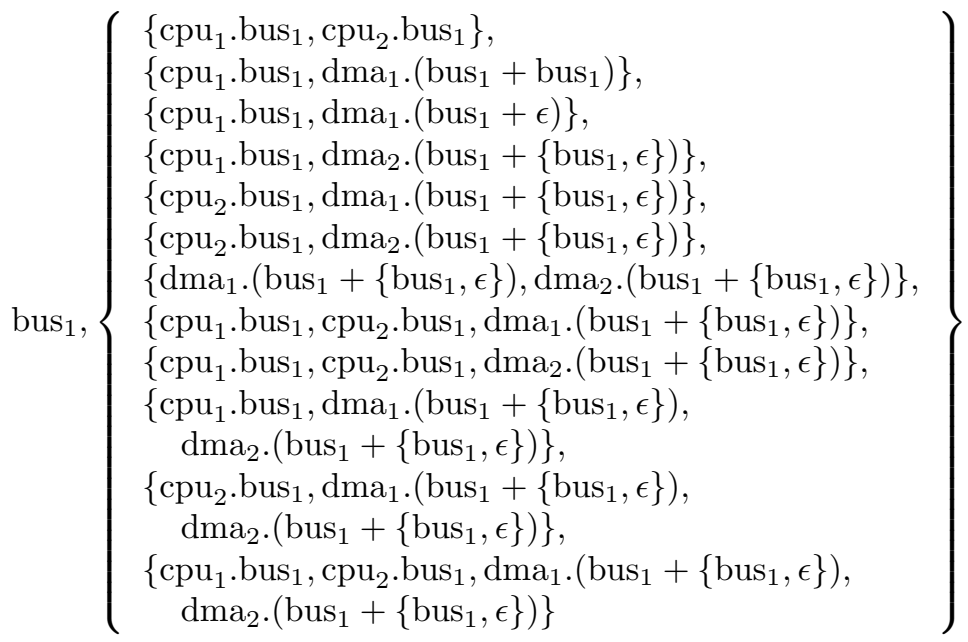




\subsubsection{2-Interference channels}

Focusing on 1-interference channels is unfortunately insufficient because of the double branches of transactions issued by non smart transactions. Indeed, those branches can conflict on two components: one per branch.

Example 8 Let us consider the architecture of Figure 4. The two non smart initiators conflict on $b_{1}$ and $c_{1}$. None of them can be excluded in the analysis.

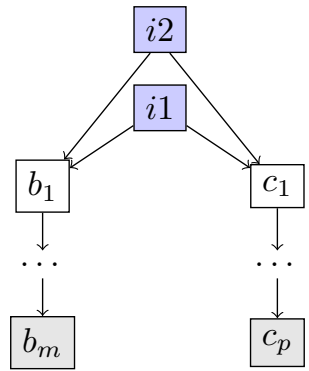

Figure 4: Architecture $P_{3}$

Thus, two components can be accessed in parallel by all transactions. We must then define 2-interference channels. There is no 3 or more-interference channels because the maximal number of branches per transaction is 2 .

Definition 12 (2-Interference channel) For an architecture $P=(\mathcal{C}, \rightarrow)$, a 2-interference channel is defined as

$$
\left(c_{1}, c_{2}, \bigcup_{n} T R_{2}^{n}\right)
$$

with $c_{1}, c_{2} \in O \cup T, c_{1} \neq c_{2}$ and

$$
T R_{2}^{n}=\bigcup\left\{\begin{aligned}
\left\{t r_{1}, \ldots,\right. & \left.t r_{n}\right\} \in \mathbb{T} \text { rans } \\
\forall i, & \left(t l\left(\operatorname{proj}_{1}\left(t r_{i}\right)\right)=c_{1} \wedge t l\left(\operatorname{proj}_{2}\left(t r_{i}\right)\right)=c_{2}\right) \\
\wedge \forall i \neq j, & h d\left(t r_{i}\right) \neq h d\left(t r_{j}\right) \\
& \wedge \operatorname{pred}\left(\operatorname{proj}_{1}\left(t r_{i}\right)\right) \neq \operatorname{pred}\left(\operatorname{proj}_{1}\left(t r_{j}\right)\right) \\
& \wedge \operatorname{pred}\left(\operatorname{proj}_{2}\left(t r_{i}\right)\right) \neq \operatorname{pred}\left(\operatorname{proj}_{2}\left(t r_{j}\right)\right)
\end{aligned}\right\}
$$

Example 9 In $P_{2}$, depicted in Figure 2, there is a unique 2-interference channel occurring on the buses.

$$
\begin{aligned}
& \operatorname{bus}_{1}, \operatorname{bus}_{2},\left\{\left\{\mathrm{dma}_{1} \cdot\left(\mathrm{bus}_{1}+\mathrm{bus}_{2}\right), \mathrm{dma}_{2} \cdot\left(\mathrm{bus}_{1}+\mathrm{bus}_{2}\right)\right\}\right\} \\
& \begin{array}{cc}
c p u_{1} \stackrel{F_{1}}{ } \\
d m a_{1} \stackrel{F_{1}}{\text { pcie } \leftrightarrow L_{3}} \quad \text { pcie } \leftrightarrow L_{3} \\
\hline
\end{array} \\
& {\begin{array}{rcc}
\text { (IO transfer for } \left.F_{1}\right) & F_{2} & F_{2} \\
{ } \leftrightarrow L_{3}
\end{array}} } & \text { pcie } \leftrightarrow L_{3} \\
& \hline m a_{2} &
\end{aligned} }
\end{array}
$$

(IO transfer for $F_{2}$ )

Figure 5: An execution scheme for architecture $P_{1}$

Example 10 There is no 2-interference channel in the $P_{1}$ architecture. This means that, with our approach, analyzing the severity of the interference caused by the 11 transactions listed in example 6 is sufficient, compared to the 7224 test classes of the initiator target model (see example 3). This reduction comes from a better modeling of the platform and from symmetry properties. The severity of the interference is evaluated with respect to the expected behavior of the software functions hosted by the platform. 
To illustrate this, let us consider the following time-triggered execution scheme depicted in figure 5:

(1) $c p u_{1}$ (resp. cpu $u_{2}$ ) hosts a software function $F_{1}$ (resp. $\left.F_{2}\right)$;

(2) memory mem (resp. mem $_{2}$ ) is dedicated to $F_{1}$ (resp. $F_{2}$ ), meaning that $F_{1}$ never tries to access mem $_{2}$ and, conversely, $F_{2}$ never tries to access mem $m_{1}$;

(3) $d m a_{1}$ (resp. dma $a_{2}$ ) manages input/output transfers of $F_{1}$ (resp. $F_{2}$ );

(4) input/output data are stored in $L_{3}$;

(5) $F_{1}$ and $F_{2}$ are periodically scheduled in non-overlapping time windows; and (6) $d m a_{1}$ (resp. dma $a_{2}$ ) is only activated by $F_{1}$ (resp. $F_{2}$ ).

Then, among the 11 transactions listed in example 6 , only the second one $\left(\left\{\mathrm{cpu}_{1}\right.\right.$.bus, $\left.\left.\mathrm{dma}_{1} \cdot(\mathrm{bus}+\mathrm{bus})\right\}\right)$ and the fifth one $\left(\left\{\mathrm{cpu}_{2}\right.\right.$. bus, dma $\left.\left.\mathrm{dm}_{2} \cdot(\mathrm{bus}+\mathrm{bus})\right\}\right)$ can occur. The potential interference caused by the other nine transactions are avoided by the execution scheme. Therefore, to meet the certification requirements it is sufficient to evaluate the WCET of $F_{1}$ (resp. $F_{2}$ ) and the WCET of the dma $a_{1}$ (resp. dma $a_{2}$ ) transfers with the interference caused by the second (resp. fifth) transaction. These interference will be said to be acceptable if these WCETs are smaller than the corresponding time windows planned by the execution model. Otherwise, they will be said to be unacceptable.

\section{Comparison between the initiator-target model and PHYLOG model}

The Phylog model can be seen as the definition of equivalence classes for the initiator-target model.

Definition 13 (Interference-free test classes) Some test classes listed in $T C_{\infty}^{P}$ do not lead to an interference channel:

1. Single test classes, as they do not generate any conflict,

2. Test classes tc such that phy $(t c)=\left(t r_{1}, \ldots, t r_{n}\right), n>1$ and $\operatorname{shared}(p h y(t c))=\emptyset$, that is, no component is shared by the $n$ transactions.

Test classes satisfying one of these two rules are said to be interference-free.

Example 11 Let us consider $P_{2}$ once again. The test class $\left\{\left(\mathrm{cpu}_{1}, \mathrm{mem}_{1}\right),\left(\mathrm{dma}_{1}, \mathrm{pcie}, \mathrm{L} 3\right)\right\}$ is interferencefree: no component is shared by the two transactions (rule 2 of the definition). However, $\left\{\left(\mathrm{cpu}_{1}, \operatorname{mem}_{1}\right)\right.$, $\left.\left(\mathrm{dma}_{2}, \mathrm{pcie}, \mathrm{mem}_{2}\right)\right\}$ is not interference-free, since bus $\mathrm{b}_{1}$ is shared by the two transactions.

Let us note that a transaction, as depicted in Figure 3 , defines a partial order relation over the language for the transactions:

Definition 14 For an architecture $P=(\mathcal{C}, \rightarrow)$ and a transaction $t r=i \cdot\left(\left(a_{1} \ldots a_{n} \cdot b_{1} \ldots b_{m}\right)+\left(a_{1} \ldots a_{n}\right.\right.$. $\left.c_{1} \ldots c_{p}\right)$ ), let us define the relation $<_{\text {tr }}$ over $\lg (\operatorname{tr})$ as:

- if $\left(a_{1} \ldots a_{n}\right) \neq \epsilon$, then $\forall i=1 \ldots n-1, a_{i}<_{t r} a_{i+1}$

- if $\left(a_{1} \ldots a_{n}\right) \neq \epsilon$, then $a_{n}<_{t r} b_{1}$

- if $\left(a_{1} \ldots a_{n}\right) \neq \epsilon$ and if $\left(c_{1} \ldots c_{p}\right) \neq \epsilon$, then $a_{n}<_{t r} c_{1}$

- $\forall i=1 \ldots m-1, b_{i}<_{t r} b_{i+1}$

- if $\left(c_{1} \ldots c_{p}\right) \neq \epsilon$, then $\forall i=1 \ldots p-1, c_{i}<_{t r} c_{i+1}$

$<_{\text {tr }}$ is the order generated by the oriented paths followed by the transaction.

Proposition 4 Let $P=(\mathcal{C}, \rightarrow),<_{P}$ is the partial order (see proposition 2), $t c=\left\{t r_{1}, \ldots, t r_{n}\right\} a$ PHYLOG test class, and $S=\operatorname{shared}(t c)$ the set of components shared by all the transactions $\operatorname{tr}_{i}$. Let

$$
\min \left(S,<_{P}\right)=\left\{\alpha \in S \mid \forall \beta \in S, \text { either } \alpha<_{P} \beta \text { or } \neg\left(\beta<_{P} \alpha\right)\right\}
$$

be the set of smallest components in $S$ for $<_{P}$. Then

- either $\min \left(S,<_{P}\right)=\emptyset$, 
- or $\min \left(S,<_{P}\right)=\{\alpha\}$ and $\forall t r_{i}, \forall \beta \in S, \alpha<_{t r_{i}} \beta$ (meaning $\alpha$ is the first component crossed by all $\left.t r_{i}\right)$

- or $\min \left(S,<_{P}\right)=\{\alpha, \beta\}$ and $\forall t r_{i}, \forall \gamma \in S, \alpha<_{t r_{i}} \gamma \vee \beta<_{t r_{i}} \gamma$ (meaning $\alpha$ (resp. $\beta$ ) is the first crossed in some branches in which $\beta$ (resp. $\alpha$ ) is not involved, as $b_{1}$ and $c_{1}$ in Figure 4).

Proof 2 Let us consider $\operatorname{tr}_{1}=i \cdot\left(\left(a_{1} \ldots a_{m} \cdot b_{1} \ldots b_{p}\right)+\left(a_{1} \cdot a_{m} \cdot c_{1} \ldots c_{q}\right)\right)$ of tc with $b_{j} \neq c_{k}$ for all $j, k$. Remember that $S=\operatorname{shared}(t c)=\lg \left(\operatorname{tr}_{1}\right) \cap \ldots \cap \lg \left(\operatorname{tr}_{n}\right)$. Then $S \subset\left\{a_{1}, \ldots, a_{m}, b_{1} \ldots, b_{p}, c_{1}, \ldots, c_{q}\right\}$. Let us consider 5 cases:

- case 1: $S=\emptyset$. Then $\min \left(S,<_{P}\right)=\emptyset$. Meaning tc is interference-free.

- case 2: $S \cap\left\{a_{1}, \ldots a_{m}\right\} \neq \emptyset$. Then $\exists k, \min \left(S,<_{t r_{1}}\right)=\left\{a_{k}\right\}$. Moreover, $\min \left(S,<_{P}\right)=\min \left(S,<_{t r_{1}}\right.$ )$=\left\{a_{k}\right\}$.

- case 3: $S \subset\left\{b_{1}, \ldots b_{p}\right\}$. Then $\exists k, \min \left(S,<_{t r_{1}}\right)=\left\{b_{k}\right\}$. Then $\min \left(S,<_{P}\right)=\min \left(S,<_{t r_{1}}\right)=\left\{b_{k}\right\}$.

- case 4: $S \subset\left\{c_{1}, \ldots c_{q}\right\}$. Then $\exists k, \min \left(S,<_{t r_{1}}\right)=\left\{c_{k}\right\}$. Then $\min \left(S,<_{P}\right)=\min \left(S,<_{t r_{1}}\right)=\left\{c_{k}\right\}$.

- case 5: $S \subset\left\{b_{1}, \ldots, b_{p}, c_{1}, \ldots c_{q}\right\}$ and $S \cap\left\{b_{1}, \ldots b_{p}\right\} \neq \emptyset$ and $S \cap\left\{c_{1}, \ldots c_{q}\right\} \neq \emptyset$. Then $\exists k, l, \min \left(S,<_{t_{1}}\right.$ )$=\left\{b_{k}, c_{l}\right\}$ with $\neg\left(b_{k}<_{t r_{1}} c_{l}\right)$ and $\neg\left(b_{k}<_{t r_{1}} c_{l}\right)$. Then $\min \left(S,<_{P}\right)=\min \left(S,<_{t r_{1}}\right)=\left\{b_{k}, c_{l}\right\}$.

Example 12 For $P_{2}$ and its $\left\{\left(\mathrm{dma}_{1}, \mathrm{mem}_{1}, \mathrm{pcie}\right),\left(\mathrm{dma}_{2}, \mathrm{mem}_{2}, \mathrm{pcie}\right)\right\}$ test class, the associated transactions are

- $t r_{1}=\mathrm{dma}_{1} \cdot\left(\right.$ bus $\left._{1} \cdot \mathrm{pcie}+\mathrm{bus}_{2} \cdot \mathrm{mem}_{1}\right)$

- $t r_{2}=\mathrm{dma}_{2} \cdot\left(\mathrm{bus}_{2} \cdot \mathrm{mem}_{2}+\mathrm{bus}_{1} \cdot \mathrm{pcie}\right)$

$S=\left\{\right.$ bus $\left._{1}, \operatorname{bus}_{2}, \operatorname{pcie}\right\}$, and $\min \left(S,<_{P}\right)=\left\{\right.$ bus $\left._{1}, \operatorname{bus}_{2}\right\}$.

Definition 15 (Relation $\equiv)$ Let us define the relation $\equiv$ on the Brindejonc et al. test classes. Let $t_{1}$ and $t c_{2}$ be two test classes. Let $S_{1}=\operatorname{shared}\left(\right.$ phy $\left.\left(t c_{1}\right)\right)$ and $S_{2}=\operatorname{shared}\left(\operatorname{phy}\left(t c_{2}\right)\right)$, then

$$
\begin{gathered}
t c_{1} \equiv t c_{2} \Longleftrightarrow \\
\min \left(S_{1},<_{P}\right)=\min \left(S_{2},<_{P}\right) \wedge\left(S_{1}=\emptyset \vee h d\left(t c_{1}\right)=h d\left(t c_{2}\right)\right)
\end{gathered}
$$

Proposition 5 The relation $\equiv$ is an equivalence relationship.

Proof $3 \equiv$ is reflexive. Indeed, let tc be a test class, $\min \left(\right.$ shared $\left.(p h y(t c)),<_{P}\right)$ is defined in a unique way.

$\equiv$ is symmetric, because we only handle sets.

$\equiv$ is transitive: if $\min \left(S_{1},<_{P}\right)=\min \left(S_{2},<_{P}\right)$ and $\min \left(S_{1},<_{P}\right)=\min \left(S_{3},<_{P}\right)$ then $\min \left(S_{2},<_{P}\right)=$ $\min \left(S_{3},<_{P}\right)$. Same for $h d\left(t c_{i}\right)$.

Proposition 6 The PHYLOG interference channels are a representative of the $\equiv$ relation classes. More precisely, let $t c=\left\{\left(i_{1}, t_{1}\right) \ldots\left(i_{m}, t_{m}, t_{m}^{\prime}\right)\right\}$, let $S=\operatorname{shared}(\operatorname{phy}(t c))$.

- if $\min \left(S,<_{P}\right)=\emptyset$, there is no interference channel in PHYLOG,

- if $\min \left(S,<_{P}\right)=\{c\}$, the associated interference channel in PHYLOG is a 1-interference channel in $c, \cup_{n} T R_{1}^{n}$, i.e. $\operatorname{tr}=\left\{t r_{1}, \ldots, t r_{m}\right\} \in T R_{1}^{m}$ with $h d(t r)=h d(t c)$;

- if $\min \left(S,<_{P}\right)=\left\{c_{1}, c_{2}\right\}$, the associated interference channel in PHYLOG is a 2-interference channel $c 1, c 2, \cup_{n} T R_{2}^{n}$, i.e. $\operatorname{tr}=\left\{t r_{1}, \ldots, t r_{m}\right\} \in T R_{2}^{m}$ with $h d(t r)=h d(t c)$.

Proof 4 Case interference-free channel: all these test classes are associated to the empty set of PHYLOG.

Case $\min \left(S,<_{P}\right)=\{c\}$ (resp. $\left.=\left\{c_{1}, c_{2}\right\}\right)$ : we apply the truncation of definition 10 where the transactions stop at $a_{p}=c\left(\right.$ resp. $a_{p}=c_{1}$ and $\left.b_{l}=c_{2}\right)$ and then $\left\{t r_{1}, \ldots, t r_{m}\right\}$ is in $T R_{1}^{m}\left(\right.$ resp. $\left.T R_{2}^{m}\right)$.

\section{$5 \quad$ Experiments}

In this section, we first illustrate the IDP code supporting the interference channels computations. We then provide some experiments with IDP. 


\subsection{Coding the initiator target model}

SI, NSI, T and $O$ are represented as types. $\rightarrow$ is a predicate with two parameters.

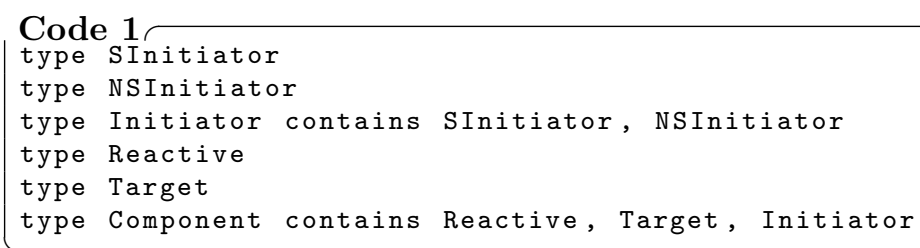

A predicate Path computes all paths from SI to $T$. For our modeling, we chose to hard code the maximal length of a path. Thus, if a path is shorter, we use $N U L L$ to complete the path. For instance, in $P_{1}$, if the maximal length is 4 , the path from $C P U_{1}$ to $m_{e} m_{1}$ is represented as $P a t h\left(C P U_{1}\right.$, interconnect, mem $\left._{1}, N U L L\right)=$ true.

Code 2

!i[Initiator]: !x1[Component]: !x2[Component]:

! $\mathrm{x} 3[$ Component]: Path $(i, \mathrm{x} 1, \mathrm{x} 2, \mathrm{x} 3)<-$

$(\operatorname{Edge}(i, x 1)$ \& $\operatorname{Edge}(x 1, x 2)$ \& $\operatorname{Edge}(x 2, x 3))$.

A predicate SingleTestCase computes the single test cases. Again, the length is imposed to 3, because of the non smart initiator. The test case $C P U_{1} \rightsquigarrow m_{1}$ is represented as SingleTestCase $\left(C P U_{1}, m_{1} m_{1}\right.$, $N U L L)=$ true.

Code 3

! i [NSInitiator]: !t1 [Target]: !t2[Target]:

SingleTestCase $(i, t 1$, t2) <-

?x[Component]: ?z[Component]: ?v[Component]:

?y[Component]: ?h[Component]: ?1[Component]:

(Path(i,x,t1,z) | Path(i,x,z,t1) | Path(i,t1,z,v))

\& $(\operatorname{Path}(i, y, t 2, h) \mid \operatorname{Path}(i, y, h, t 2)$

I Path (i,t2,h,l)).

Finally, there is one predicate per $T C_{n}^{P}$. For instance, test cases of size 2 are coded by CoupleTestCase.

Code 4

!i1[NSInitiator]: !t1[Target]: !t2[Target]:

! i2 [NSInitiator]: !t 3 [Target]: !t 4 [Target]:

CoupleTestCase(i1,t1, t2, i2, t3,t4) <-

(SingleTestCase (i1, t1, t2) \&

SingleTestCase $(i 2, t 3, t 4)$ \& (i1<i2)).

\subsection{Coding the Phylog model}

We compute an intermediate predicate to combine two transactions composed of a single branch each. Trans2, still for a maximal of 3 components in a path, is defined below.

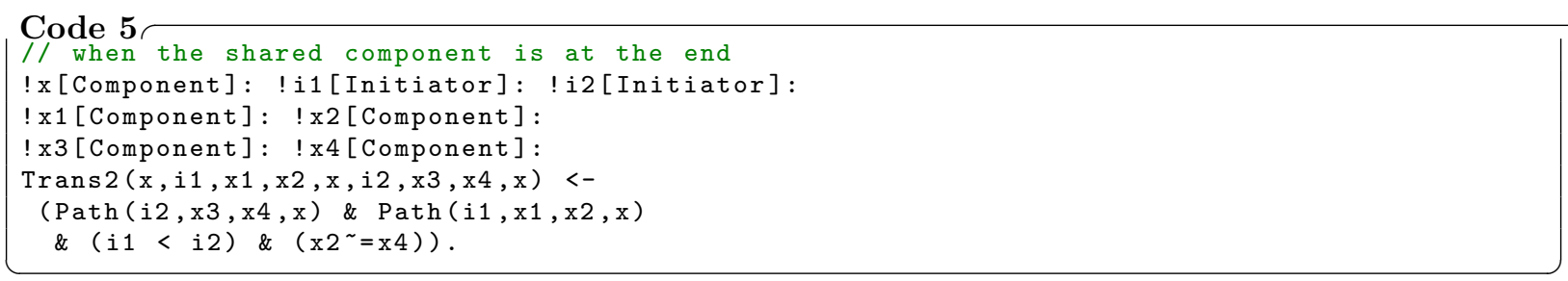

There is one predicate per $T R_{1}^{n}$. For instance, $T R_{1}^{2}$ is coded by combining the two branches of a pair 


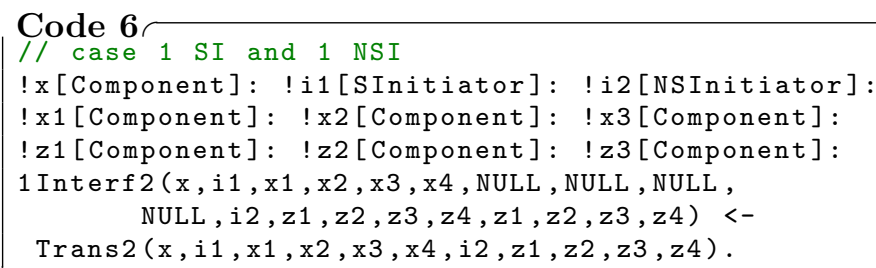

There is also one predicate per $T R_{2}^{n}$. The code for $T R_{2}^{2}$ is given below.

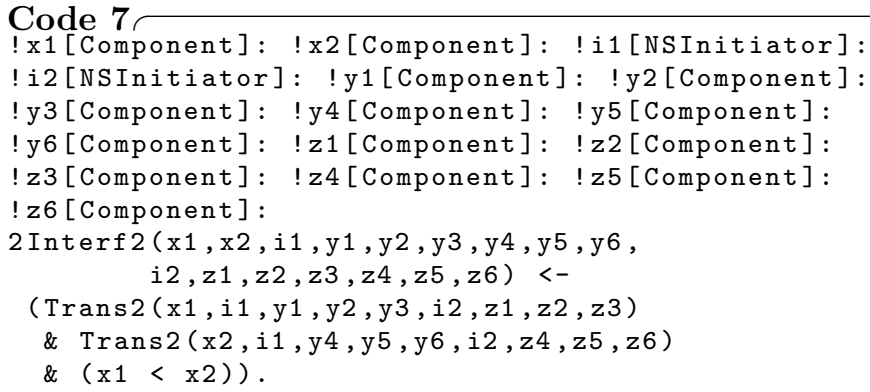

\subsection{Some results}

IDP computes the predicates of the previous examples in less than a second. Increasing the number of smart initiators, non-smart initiators, targets, or intermediary components does not appear to increase the execution time. Even when modeling a Kalray MPPA [8] compute cluster, composed of 16 cores, 32 intermediary components, and 16 targets, resolution of those predicates (exposing a total of 1920 interference channels) is still completed in sub-second times.

To ensure their correctness, we have also used IDP to compute the $\equiv$ relation classes of the aforementioned examples, and found them to be compliant with the propositions made in this paper.

\section{Related Work}

Interference analysis in multi-core processors has received significant attention in recent years. A first class of these works focuses on the impact of shared hardware resource contention on the execution time of software application hosted by the processor. For instance, 9] considers a multi-core architecture composed of a single bus providing access to a shared memory, and it proposes a method to determine an upper bound on the number of bus requests that software tasks can generate in a given time interval. Both [5] and [12] focus on measurement techniques based on dedicated stressing benchmarks and hardware monitors to characterize the architecture and the shared resources that can cause interference between software applications.

A second class of works focuses on methods to avoid interference. For instance, 2] proposes a contention-free execution framework to execute automotive software application on many-core platforms. [19] proposed a similar approach which relies both on a development work-flow, and the use of an execution model defined as a set of rules to be followed by the designer and asserted through the run-time in order to enforce specific behaviors. Both [2] and [19] target a TDMA execution model, and use a Constraint Programming formulation to find an optimal time-triggered schedule on each core.

In order to tackle multi-core aeronautics certification-related issues, several projects have been funded. One of the first was MULCORS [13, which clearly identified the need to change and adapt the current certification standard. Since then, several attempts at precisely defining such new recommendation have been done, such as the Multi-core Certification Review Item (MCP-CRI) [11. In other parts of the MCPCRI, [15] proposed definitions for interference channels, interference sources, and interference targets, and they proposed a process to reduce the number of interference. A more recent work proposed by 1$]$ tried to adapt the MCP-CRI certification objectives to COST MCP architectures. For that purpose, they showed that the MCP-CRI objectives can be grouped into three high level principles: (1) determining the final configuration, (2) managing interference channels, and (3) verifying the use of shared resources. They 
showed, through a particular case study (the Freescale P4080 processor), that the second objective (managing interference channels) highly depends on detailed information about the behavior of the resources. And they showed that predicting interference on a COTS multi-core architecture is a very challenging task because of the amount of required information. A way to help the certification application to master the complexity of the architecture is then to use a formal model of the architecture and a formal analysis method to explore the set of interference channels. Such is the aim of our contribution.

\section{Conclusion}

In this paper, we have formally defined the initiator-target model and compared it with the PHYLOG approach. Our representation requires more details on the internal of the platform but offers a more practical size description. Our work was supported with IDP tool.

In the future, we will apply our model to other multi-cores and extend our model to many-core platforms. We will also measure the gains of going deeper in the description of the architecture.

\section{References}

[1] Irune Agirre, Jaume Abella, Mikel Azkarate, and Francisco Cazorla. On the Tailoring of CAST$32 \mathrm{~A}$ Certification Guidance to Real COTS Multicore Architectures. In 12th IEEE International Symposium on Industrial Embedded Systems (SIES'17), 2017.

[2] Matthias Becker, Dakshina Dasari, Borislav Nicolic, Benny Åkesson, Vincent Nélis, and Thomas Nolte. Contention-free execution of automotive applications on a clustered many-core platform. In 28th Euromicro Conference on Real-Time Systems, July 2016.

[3] Pierre Bieber, Frédéric Boniol, Youcef Bouchebaba, Julien Brunel, Claire Pagetti, Olivier Poitou, Thomas Polacsek, Luca Santinelli, and Nathanaël Sensfelder. A model-based certification approach for multi/many-core embedded systems. In 9th European Congress on Embedded Real Time Software and Systems (ERTS 2018), 2018.

[4] Pierre Bieber, Frédéric Boniol, Guy Durrieu, Olivier Poitou, Thomas Polacsek, Virginie Wiels, and Ghilaine Martinez. MIMOSA: Towards a model driven certification process. In Proc. 8th Int. Congress on Embedded Real Time Software and Systems (ERTS'16), 2016.

[5] Jingyi Bin, Sylvain Girbal, Daniel Gracia Perez, Arnaud Grasset, and Alain Merigot. Studying co-running avionic real-time applications on multi-core cots architectures, 022014.

[6] Vincent Brindejonc and Anthony Roger. Avoidance of dysfunctional behaviour of complex cots used in an aeronautical context. In 19eme Congrès de Maîtrise des Risques et Sûreté de Fonctionnement, 2014.

[7] Certification Authorities Software Team. Multi-core Processors - Position Paper. Technical Report CAST 32-A, November 2016.

[8] Kalray Corporation. The MPPA hardware architecture, 2012.

[9] Dakshina Dasari and Vincent Nelis. An analysis of the impact of bus contention on the wcet in multicores. In Proceedings of the 2012 IEEE 14th International Conference on High Performance Computing and Communication 85 2012 IEEE 9th International Conference on Embedded Software and Systems, HPCC '12, pages 1450-1457, Washington, DC, USA, 2012. IEEE Computer Society.

[10] Broes de Cat, Bart Bogaerts, Maurice Bruynooghe, and Marc Denecker. Predicate logic as a modelling language: The IDP system. CoRR, abs/1401.6312, 2014.

[11] EASA (European Aviation Safety Agency). The Use of Multi-Core Processors in Safety-Critical Applications - CRI, 2016.

[12] Sylvain Girbal, Jingyi Bin, Daniel Gracia Perez, and Alain Merigot. Using monitors to predict co-running safety-critical hard real-time benchmark behavior. In Conference on Information and Communication Technology for Embedded Systems (ICITES'14), 012014. 
[13] Xavier Jean, Marc Gatti, Guy Berthon, and Marc Fumey. MULCORS-Use of Multicore Processors in airborne systems. European Aviation Safety Agency, Industrial report December, 2012.

[14] Xavier Jean, Laurence Mutuel, and Vincent Brindejonc. Assurance methods for cots multi-cores in avionics. In 35th Digital Avionics Systems Conference (DASC'16), 2016.

[15] Xavier Jean, Laurence Mutuel, Didier Regis, Hélène Misson, Guy Berthon, and Marc Fumey. White Paper on Issues Associated with Interference Applied to Multicore Processors, 2016. Retrieved from http://www.faa.gov/aircraft/air_cert/design_approvals/air_ software/media/SDS_D0005_White_Paper.pdf

[16] Jean-Yves Le Boudec and Patrick Thiran. Network Calculus: A Theory of Deterministic Queuing Systems for the Internet. Springer-Verlag, Berlin, Heidelberg, 2001.

[17] Laurence Mutuel, Xavier Jean, and Vincent Brindejonc. Investigation of error types associated with failures in multicore processors. In 20eme Congrès de Maîtrise des Risques et Sûreté de Fonctionnement, 2016.

[18] Laurence Mutuel, Xavier Jean, Vincent Brindejonc, Anthony Roger, Thomas Megel, and E. Alepins. Assurance of Multicore Processors in Airborne Systems, 2017.

[19] Quentin Perret, Pascal Maurère, Éric Noulard, Claire Pagetti, Pascal Sainrat, and Benoît Triquet. Temporal isolation of hard real-time applications on many-core processors. In 22th IEEE Real-Time and Embedded Technology and Applications Symposium (RTAS'16), April 2016.

[20] Reinhard Wilhelm, Jakob Engblom, Andreas Ermedahl, Niklas Holsti, Stephan Thesing, David Whalley, Guillem Bernat, Christian Ferdinand, Reinhold Heckmann, Tulika Mitra, Frank Mueller, Isabelle Puaut, Peter Puschner, Jan Staschulat, and Per Stenström. The worst-case executiontime problem - overview of methods and survey of tools. ACM Transactions Embedded Computing Systems, 7(3):36:1-36:53, May 2008.

[21] Reinhard Wilhelm and Jan Reineke. Embedded systems: Many cores - many problems. In 7th IEEE International Symposium on Industrial Embedded Systems (SIES'12), pages 176-180, 2012. 